[0212-7199 (2004) 21: 11; pp 533-539] ANALES DE MEDICINA INTERNA Copyright (C) 2004 ARAN EDICIONES, S.L.

AN. MED. INTERna (Madrid) Vol. 21, N. ${ }^{\circ} 11$, pp. 533-539, 2004

\title{
Espondilodiscitis infecciosas en un área sanitaria gallega, 1983-2003
}

\author{
N. GÓMEZ RODRÍGUEZ, Y. PENELAS-CORTÉS BELLAS', J. IBÁÑEZ RUÁN, \\ M. GONZÁLEZ PÉREZ, M. L. SÁNCHEZ LORENZO²
}

Servicio de Reumatología. ${ }^{1}$ Unidad de Codificación de Diagnósticos del Centro Médico POVISA. Vigo. Pontevedra. ${ }^{2}$ Complejo Hospitalario Universitario de Santiago de Compostela. A Coruña

INFECTIOUS SPONDYLODISCITIS IN A HEALTH AREA OF GALICIA (SPAIN) FROM 1983-2003

\section{RESUMEN}

Objetivo: Estudiar las características clínicas y epidemiológicas de las espondilodiscitis infecciosas (EI) diagnosticadas en nuestra área sanitaria durante los últimos 20 años.

Material y métodos: Análisis retrospectivo de las historias de los pacientes adultos con EI tuberculosas (EIT) y de otra etiología (EINT) cuyo diagnóstico se realizó entre el 1 de enero de 1983 y el 31 de diciembre de 2003. Se incluyeron aquellos casos con cuadro clínico y radiológico compatible asociado a la recuperación del microorganismo en dos o más hemocultivos y / o del material obtenido del foco de EI. También se consideró probado el diagnóstico de EIT si existían granulomas caseificantes típicos en las biopsias vertebrales o de focos extravertebrales concomitantes.

Resultados: Se recogieron 17 EIT y 22 EINT. La edad de los pacientes fue inferior en las EIT $(43,5 \pm 24,6$ vs 52,0 $+15,2$ años; media \pm desviación estándar). Los varones predominaron en ambos grupos, sobre todo en las EINT ( $82 \%$ vs $57 \%$, p < 0,0001). El intervalo entre el inicio de los síntomas y el diagnóstico fue más corto en las EINT ( $3,9 \pm 3,2$ vs 16,4 $\pm 15,2$ semanas, $p=0,005)$. En siete $(41 \%)$ de los pacientes con EIT existían otros focos tuberculosos activos. En el 90\% de las EINT se identificaron potenciales puertas de entrada para los microorganismos, destacando las intervenciones en la columna vertebral $(9 / 22,41 \%)$. Se registraron factores predisponentes generales en 3 EIT y 4 EINT. En las EINT fueron más frecuentes la leucocitosis $(41 \%$ vs $12 \%, \mathrm{p}<0,001)$ y la fiebre ( $41 \%$ vs $24 \%, \mathrm{p}=0,0003)$, mientras los déficit neurológicos en el momento del diagnóstico predominaron en las EIT (24\% vs $9 \%, \mathrm{p}=$ $0,01)$. Todas las EINT fueron monobacterianas, destacando Staphylococcus aureus $(14 / 22,64 \%)$, seguido del género Streptococcus $(6 / 22,27 \%)$. Klebsiella pneumoniae y Proteus mirabilis causaron las dos EINT restantes. Las EIT requirieron más intervenciones para descompresión mielorradicular y / o drenaje de abscesos paravertebrales ( $29 \%$ vs $18 \%$; $\mathrm{p}=$ $0,0027)$ y presentaron más secuelas neurológicas $(24 \%$ vs $14 \%$; $\mathrm{p}=$ $0,008)$. La incidencia global estimada de EI en nuestro sector sanitario fue de 2,2 casos $/ 10^{5}$ habitantes / año, de los que 1,73 corresponderían a EINT.

Conclusiones: Las EI son poco frecuentes y predominan en varones. Durante la última década se ha producido un notable incremento de las EINT, con participación significativa $(41 \%)$ de las postquirúrgicas. Las EIT sufren una mayor demora en el diagnóstico y ello se asocia a mayor prevalencia de secuelas.

PALABRAS CLAVE: Espondilodiscitis infecciosa. Osteomielitis vertebral. Tuberculosis. Epidemiología.

\section{ABSTRACT}

Objective: To study the clinical-epidemiological characteristics of infectious spondylodiscitis (IS) in adults of our health area in the late 20 years.

Material and methods: We performed a retrospective analysis of the medical records of adult patients with a diagnosis of both, tuberculous (TS) and non tuberculous spondylodiscitis (NTS), between January 1983 and December 2003. The diagnosis was made when compatible clinicalradiological picture were present in association with at least two positive blood cultures and / or micro-organism recovery from vertebral samples. Additionally, TS was diagnosed when biopsy showed typical caseating granulomas from vertebral or extra-vertebral lesions.

Results: 17 TS and 22 NTS were identified. The mean age was lower in patients with TS than in NTS $(43.5 \pm 24.6$ vs $52.0 \pm 15.2$ years; mean \pm SD). Patients were predominately males in both. TS $(57 \%)$ and NTS (82\%; $p<0001)$. The time between the onset of symptoms and diagnosis was longer in TS (16,4 $\pm 15,2$ weeks) than in NTS (3.9 \pm 3.2 weeks), $p=$ 0.005 . Seven (41\%) of the 17 patients with TS had active extra-vertebral tuberculosis. A source of infection was presumed in 20 NTS (90\%), mainly surgical spinal procedures (9/22.41\%). In three TS and four NTS one o more predisposing factors were observed. The patients with NTS presented a higher prevalence of fever (41\% vs $24 \%$; $p=0.0003)$ and leucocytosis $(41 \%$ vs $12 \%$; $p<0.001)$, but less neurological impairment $(9 \%$ vs $21 \% p=0,01)$. None patient with NTS presented mixed infection and Staphylococcus aureus was the main pathogen (14/22.64\%) follows by Streptococcus $s p(6 / 22.27 \%)$. Klebsiella pneumoniae and Proteus mirabilis were the remain causative agents. Spinal cord decompression and surgical drainage of abscess were performed in five patients $(24 \%)$ with TS and four patients with NTS (18\%), $p=0.0027)$. Neurological sequels were more common in the patients with TS $(24 \%$ vs $14 \%, p=$ $0.008)$. The global incidence of IS was 2.2 cases / $10^{5}$ inhabitants / year, which 1.73 cases are NTS.

Conclusions: The IS are a unusual disease what occurs predominantly in male patients. In the last 10 years, a increasing prevalence of NTS was observed, with high contribution (41\%) of post-surgical cases. Diagnostic delay is greater in patients with TS and this condition was associated with more neurological sequels.

KEY WORDS: Infectious spondylodiscitis. Vertebral osteomyelitis. Tuberculosis. Epidemiology.

Gómez Rodríguez, N, Penelas-Cortés Bellas Y, Ibáñez Ruán J, González Pérez M, Sánchez Lorenzo ML. Espondilodiscitis infecciosas en un área sanitaria gallega, 1983-2003. An Med Interna (Madrid) 2004; 21: 533-539. 


\section{INTRODUCCIÓN}

Se denomina espondilodiscitis infecciosa $(E I)$ al proceso de colonización y ulterior infección del espacio discal y de los cuerpos vertebrales adyacentes, ya que, en el momento del diagnóstico, ambos suelen estar involucrados $(1,2)$. La infección independiente del disco (discitis) o de una vértebra (espondilitis) es mucho menos habitual. Los microorganismos, mayoritariamente bacterias, suelen acceder a estas estructuras por vía hematógena (2-7). Durante los últimos 20 años se ha señalado una disminución de las espondilodiscitis tuberculosas (EIT) y un incremento de las ocasionadas por otros microorganismos (EINT). Entre los factores implicados en estos cambios etiológicos cabe considerar la difusión de la adicción a drogas por vía parenteral (ADVP), las maniobras instrumentales en el sistema genitourinario y la cateterización del árbol vascular (8), el incremento de los pacientes con enfermedades crónicas, trasplantados e inmunosuprimidos $(9,10)$ y un mayor número de intervenciones sobre la columna vertebral.

Pese a su escasa frecuencia, se han publicado un número sustancial de series centradas en las manifestaciones clínicas, diagnóstico y tratamiento, tanto de las EIT (1,2,11-14) como de las EINT $(1,2,4,6,9,12)$. Sin embargo, en nuestra comunidad autónoma, apenas disponemos de información epidemiológica y clínica de las EI, motivo por el que hemos analizado los casos diagnosticados en nuestro hospital durante los últimos 20 años (1983-2003).

\section{MATERIAL Y MÉTODOS}

Hemos procedido a revisar en nuestro hospital, de forma retrospectiva y protocolizada, las historias de todos los pacientes diagnosticados de EI, tanto tuberculosas como de otra etiología, durante el periodo comprendido entre el 1 de enero de 1983 hasta el 31de diciembre de 2003. Se excluyeron las historias de seis pacientes que no cumplían los requisitos para su inclusión. Estos consistieron en un cuadro clínico y radiológico compatible asociado a la recuperación del microorganismo en dos o más hemocultivos y/o del material obtenido del foco de EI. También se consideró probada la etiología tuberculosa ante la presencia de granulomas caseificantes típicos en las biopsias vertebrales o de otros focos extravertebrales concomitantes junto con la respuesta favorable al tratamiento tuberculostático (1). De cada historia se recogieron los siguientes datos: edad, sexo, antecedentes personales, posible puerta de entrada, manifestaciones clínicas y tiempo transcurrido desde su inicio hasta el diagnóstico, resultados de los análisis y de los medios de diagnóstico por la imagen, estudios microbiológicos y técnica para la obtención de las muestras, tratamiento, complicaciones y evolución.

El análisis estadístico de la asociación entre variables cualitativas se realizó con la prueba de Chi cuadrado o, cuando las condiciones lo exigieron, la prueba exacta de Fisher. Para las variables continuas se utilizó la prueba de la t de Student. Se consideraron significativos los valores de $\mathrm{p}<0,05$.

Mediante Medline Embase-plus revisamos la literatura médica sobre las espondilodiscitis infecciosas en el periodo 1980-2003, ambos inclusive, utilizando las siguientes palabras clave: osteomielitis vertebral, espondilodiscitis infecciosa, espondilitis infecciosa y espondilodiscitis bacteriana.

\section{RESULTADOS}

Se recogieron 17 EIT y 22 EINT cuya distribución durante el periodo de estudio se expone en la figura 1. Todos los pacientes fueron caucasianos y de nacionalidad española, con una mediana de edad inferior en las EIT $(43,5 \pm 24,6$ versus $52,0 \pm 15,2 ;$ media \pm desviación estándar). Hubo un predominio de varones en ambos grupos, sobre todo en las EINT (82\% vs $57 \%$, p < 0,0001). El 68\% de los pacientes con EINT tenían una edad comprendida entre los 40 y 70 años (Fig. 2). El intervalo entre el inicio de los síntomas y el diagnóstico fue significativamente superior en las EIT $(16,4 \pm 15,2$ vs 3,9 $\pm 3,2$ semanas, $p=0,005)$.
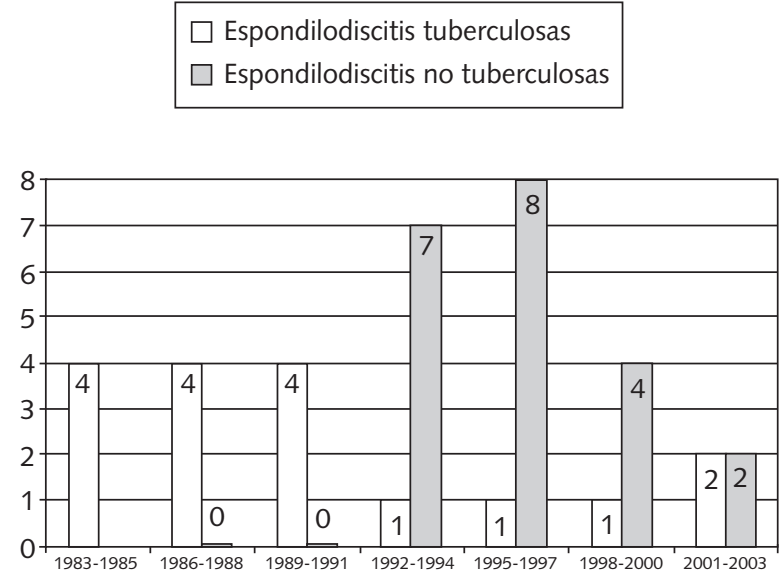

Fig. 1. Distribución de los pacientes en el periodo de estudio (19832003).
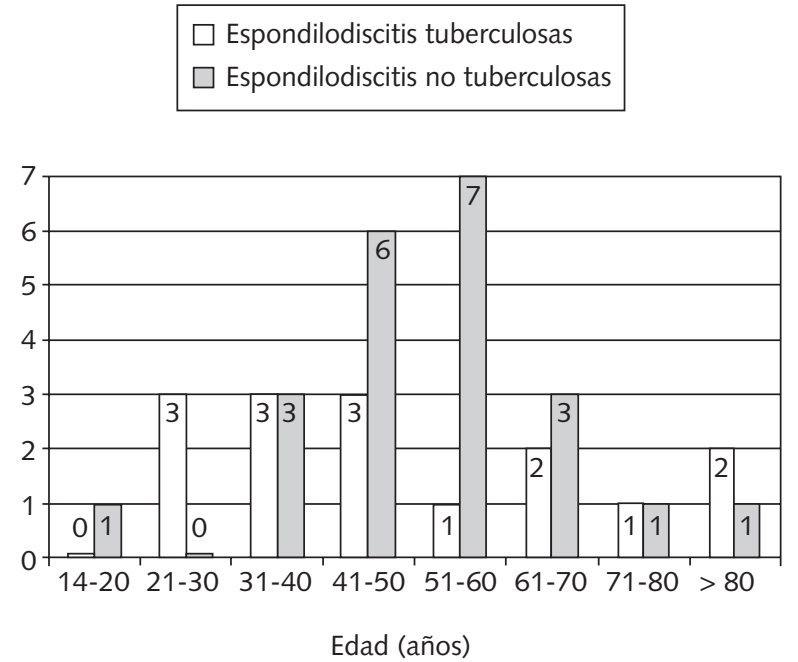

Fig. 2. Distribución etaria de los pacientes con espondilodiscitis infecciosas.

Entre los pacientes con EIT, dos sufrían una hepatitis B crónica y otro recibía esteroides debido a una enfermedad de Crohn; además, en siete $(41 \%)$ se identificaron otros focos tuberculosos activos: dos tuberculosis pulmonares y cinco pleuritis tuberculosas. En el $90 \%$ de los pacientes con EINT 
se hallaron potenciales puertas de entrada para los microorganismos: intervenciones en la columna vertebral (9), histerectomía (2), adicción a drogas por vía parenteral (ADVP) (2), infección urinaria (2), piorrea (1), forúnculo (1) y úlcera plantar (1). Este última correspondía al único paciente diabético de nuestra serie que, además, presentaba una hepatopatía crónica por virus C. Uno de los dos pacientes con ADVP cumplía criterios para el diagnóstico de síndrome de inmunodeficiencia adquirida.

Todos los pacientes con EI presentaron raquialgias localizadas de ritmo inflamatorio. Durante el curso clínico de los enfermos con EIT, cuatro (24\%) tuvieron fiebre termometrada $\left(>37,7^{\circ}\right.$ en axila) frente a nueve $(41 \%)$ de los pacientes con EINT ( $p=0,0003)$. En el momento del diagnóstico, la semiología de compromiso mielorradicular fue más común en los pacientes con EIT $(24 \%$ vs $9 \%$ p < 0,02). En tres EIT $(20 \%)$ estaban involucradas cuatro vértebras contiguas, mientras que sólo objetivó afectación de más de dos vértebras consecutivas en una EINT (4,5\%). La distribución de las lesiones fue diferente para las EIT y las EINT. Así, en las EIT el segmento dorsal (6 casos) y la charnela dorsolumbar (5 casos) fueron las localizaciones más frecuentes, seguidas del segmento lumbar ( 5 casos) y el cervical (1 caso). En las EINT, la columna lumbar estuvo involucrada en 14 pacientes, seguida de la charnela lumbosacra, segmento dorsal y cervical, implicados en cuatro, tres y un paciente, respectivamente.

No hubo diferencias significativas de la VSG, que se determinó en 19 EINT y en 15 EIT $(69,6 \pm 25,9$ vs 59,8 $\pm 31,9$ $\mathrm{mm} / 1^{\mathrm{a} h}$ ). Al ingreso, la leucocitosis (recuento leucocitario $>$ $11.000 / \mathrm{mm}^{3}$ ) fue significativamente más frecuente en los enfermos con EINT (41\% vs $12 \%$; p < 0,001). El paciente con EINT y SIDA presentaba leucopenia $\left(2.530 / \mathrm{mm}^{3}\right)$. La intradermorreacción de la tuberculina fue positiva (induración $>10 \mathrm{~mm}$ ) en los 15 pacientes con EIT en quienes se realizó frente a sólo $4(18 \%)$ con EINT ( $p<00001$. Los hemocultivos fueron positivos en 9 de las 14 EINT en las que se realizaron $(64 \%)$. Las radiografías mostraron alteraciones compatibles con el diagnóstico de EI en todas las EIT y en 18 de las EINT (86\%). Como estudios de imagen complementarios se empleó la tomografía computarizada (12 EIT y 4 EINT) y / o la resonancia magnética (5 EIT y 20 EINT), que permitieron objetivar abscesos paravertebrales en el $86 \%$ de las EIT (12/14) y en el $48 \%$ de las EINT (10/21), p<0,0001. Se evidenciaron abscesos epidurales mediante resonancia magnética en una EIT y en cuatro EINT, tres de los cuales presentaban semiología de sufrimiento mielorradicular. También se efectuaron gammagrafías óseas con $\mathrm{Tc}^{99 \mathrm{~m}}$ (5 EIT y 5 EINT) y / con Galio $^{67}$ (2 EIT y 4 EINT), mostrando invariablemente hipercaptación en los focos vertebrales y, en el caso del $\mathrm{Ga}^{67}$, también en los paravertebrales.

Entre los afectados por EIT, el procedimiento de obtención de material para el diagnóstico etiológico consistió en una biopsia con trocar en cinco (29\%), biopsia abierta en cuatro (24\%) y punción aspirativa en los restantes ocho enfermos (47\%), en uno de los cuales el material se extrajo de un absceso paravertebral. Las tinciones de auramina-rodamina y / o Ziehl-Neelsen fueron positivas en 11/17 (65\%) y el cultivo en medio Löwestein-Jensen del aspirado o de muestras tisulares permitió el asilamiento de Mycobacterium tuberculosis en 15/17 (88\%). El estudio histológico mostró granulomas caseificantes típicos en los 10 casos en los que se realizó. Entre las EINT, el aislamiento bacteriano en dos o más hemocultivos constituyó el único medio para el diagnóstico etiológico en cinco enfermos (23\%); en tres (14\%) se cultivó el mismo microorganismo en hemocultivos y en el material obtenido por punción aspirativa; en siete (32\%) el diagnóstico etiológico se realizó a partir de una biopsia abierta y, finalmente, en uno de los enfermos (5\%) se el tejido se consiguió mediante biopsia con trocar.

La etiología fue monobacteriana en todos los casos, incluidos los postquirúrgicos. En las EINT, Staphylococcus aureus

TABLA I

\begin{tabular}{|c|c|c|c|}
\hline \multicolumn{4}{|c|}{ TABLA I } \\
\hline \multicolumn{4}{|c|}{ COMPARACIÓN ENTRE LAS ESPONDILODISCITIS TUBERCULOSAS Y NO TUBERCULOSAS } \\
\hline Parámetro & $\begin{array}{c}\text { Espondilodiscitis } \\
\text { tuberculosas }(n=17)\end{array}$ & $\begin{array}{c}\text { Espondilodiscitis } \\
\text { no tuberculosas }(n=22)\end{array}$ & Valor de $p$ \\
\hline$E d a d \pm D^{a}$ (años) & $43,6 \pm 24$ & $52,0 \pm 15,2$ & No significativo \\
\hline Varones & $10(53 \%)$ & $18(82 \%)$ & $<0,0001$ \\
\hline \multicolumn{4}{|l|}{ Retraso diagnóstico ${ }^{b}$} \\
\hline (Semanas) & $16,4 \pm 15,2$ & $3,9 \pm 3,2$ & 0,005 \\
\hline Enfermedades crónicas debilitantes & $3(18 \%)$ & $4(18 \%)$ & No significativo \\
\hline $\operatorname{VSG}\left(\mathrm{mm} / 1^{\mathrm{a} h}\right)$ & $59,9 \pm 31,9$ & $69,6 \pm 25,9$ & No significativo \\
\hline Leucocitosis & $13 \%$ & $38 \%$ & 0,0009 \\
\hline Fiebre (\%) & $4(24 \%)$ & $9(41 \%)$ & 0,0003 \\
\hline Abscesos paravertebrales & $12(80 \%)$ & $10(45 \%)$ & $<0,0001$ \\
\hline $\begin{array}{l}\text { Compromiso mielorradicular } \\
\text { en el momento del diagnóstico }\end{array}$ & $4(24 \%)$ & $2(9 \%)$ & $<0,02$ \\
\hline Tratamiento quirúrgico & $5(29 \%)$ & $4(18 \%)$ & $<0,003$ \\
\hline Secuelas neurológicas & $4(24 \%)$ & $3(15 \%)$ & $<0,009$ \\
\hline Deformidad vertebral residual significativa & $14(82,3 \%)$ & $5(22,7 \%)$ & $<0,025$ \\
\hline
\end{tabular}

a) DE: desviación estándar; b) tiempo transcurrido desde el inicio de los síntomas hasta el diagnóstico; c) Cifosis o escoliosis sintomática y /o aparente a la simple inspección física. 
fue el microorganismo más frecuentemente aislado (64\%), seguido del género Streptococcus (27\%). En los dos pacientes restantes, la causa de la EINT se correspondió con la de una reciente infección urinaria, Proteus mirabilis y Klebsiella pneumoniae, respectivamente. De las nueve EI posquirúrgicas, siete fueron por Staphylococcus aureus (uno de ellos meticilín-resistente) y dos fueron producidas por Streptococcus pyogenes.

En tres EINT se realizó cirugía a causa de compromiso mielorradicular, pero ninguna requirió drenaje quirúrgico de los abscesos paravertebrales, intervención que fue necesaria en tres EIT. En otros tres casos de EIT se realizó cirugía de descompresión mielorradicular, dos ellos por absceso epidural. Un paciente con EINT dorsal por S. aureus requirió cirugía para drenaje de un empiema pleural izquierdo.

Ningún paciente falleció. En los EIT fueron más frecuentes las secuelas neurológicas $(24 \%$ vs $14 \%$; p < 0,01$)$ y las deformidades vertebrales residuales como grados variables de cifosis y escoliosis ( $18 \%$ vs $5 \%$; $\mathrm{p}=0,025)$. Sólo se objetivó una recidiva, que correspondió a un paciente con EIT y se produjo a los tres años de finalizado el tratamiento.

POVISA es el centro asignado, desde 1993, a través de un concierto con el SERGAS (Servicio Gallego de Salud), a la población adulta de un área sanitaria de 127.000 habitantes de los que más de 70.000 corresponden a núcleos urbanos. Si nos ceñimos a los diagnósticos realizados a partir del 1 de enero de 1993 y hasta el 31 de diciembre de 2003 en pacientes adultos de dicho sector sanitario, (6 EIT y las 22 EINT), puede estimarse una incidencia global de 2,2 casos / 10 $0^{5}$ habitantes/año, de los que 1,73 casos corresponderían a EINT. A este mismo periodo (1993-2003) corresponden las nueve EI postquirúrgicas, que supusieron el $41 \%$ de las EINT. El número total de intervenciones sobre la columna vertebral realizadas durante este periodo en pacientes de nuestro sector sanitario fue de 1.887 , lo que implica una frecuencia de 1 caso por cada 210 operaciones $(0,47 \%)$.

En la tabla I se comparan los parámetros demográficos y clínicos más significativos de las EIT y las EINT.

\section{DISCUSIÓN}

Las EI suponen cerca del 5\% de las osteomielitis en pacientes adultos y aunque existen pocos estudios sobre su epidemiología, una reciente revisión realizada en Dinamarca, halló una incidencia promedio de 5 casos de EINT por cada millón de habitantes y año (15), notablemente inferior a la registrada en nuestra área sanitaria 17,3 casos / $10^{6}$ habitantes / año, incluso si excluimos los casos postquirúrgicos en dicho periodo (10,1 /106 habitantes / año). Nos ha llamado la atención que, desde 1983 hasta 1993, casi la totalidad de las EI recogidas fueron de etiología tuberculosa, mientras que, en la siguiente década, predominaron las EINT en una proporción de 3,68:1. Aunque es bien conocido que las mejoras en el diagnóstico y tratamiento de la tuberculosis pulmonar, que constituye el foco habitual desde el que los bacilos se diseminan por vía hematógena hacia otros asentamientos, ha contribuido a reducir la incidencia de osteomielitis tuberculosa, consideramos que la marcada disminución de las EIT a partir de 1993, fecha del concierto con el SERGAS, pudo verse favorecida por el tipo de población asignado a nuestro centro, que pasó a ser mayoritariamente urbana y con actividad laboral en los sectores industrial y de servicios. Todos nuestros pacientes fueron caucasianos y de nacionalidad española, aunque en las series recientes, las EIT en emigrantes de países subdesarrollados suponen más de la mitad de los casos recogidos $(14,16)$. En general, en las EI se aprecia un predominio de varones $(3,4,7,14)$ con un promedio cercano a $1,5: 1$, similar al que encontramos entre nuestros pacientes con EIT; no obstante, la proporción de varones en las EINT $(4,5: 1)$ supera notablemente a la reseñada por otros autores $(3,4,17)$. La mayoría de los pacientes con EINT tienen entre 40 y 70 años, con una mediana de edad en la quinta o sexta década de la vida $(1,3,4,7,15,17)$.

Casi la mitad de los pacientes con EIT, $41 \%$ en nuestra serie, presentan otros focos activos de tuberculosis, sobre todo pleural y pulmonar (1). En un porcentaje similar de pacientes con EINT se hallan posibles puertas de entrada y / o focos infecciosos $(7,17)$. Pese al carácter retrospectivo de nuestro estudio, se hallaron focos infecciosos o una potencial puerta de entrada en 18 de los 22 pacientes (81\%). Esta elevada tasa probablemente estuvo condicionada por las EINT postquirúrgicas, que supusieron el $36 \%$ del total, lo que triplica el porcentaje reseñado en otras series $(4,19,20)$. La diabetes mellitus, las hepatopatías crónicas, los tratamientos inmunosupresores y el alcoholismo son los factores predisponentes más habituales $(3,4,8,15,21)$ y suelen estar presentes en cerca de la mitad de los enfermos $(4,17,18)$. No parece que el SIDA, por sí mismo, favorezca de modo significativo el desarrollo de infecciones osteoarticulares y, entre ellas, las espondilodiscitis $(4,22)$. Sería la adicción a drogas por vía parenteral, hábito tóxico frecuente entre los enfermos con SIDA, el que predispondría a las bacteriemias y posterior colonización osteoarticular de los microorganismos. Por su parte, las alteraciones estructurales óseas del raquis, como consecuencia de infecciones previas, traumatismos o cambios artrósicos, pueden constituir un elemento focalizador (2).

El dolor axial localizado de ritmo inflamatorio es el principal síntoma de las EI, al que pueden asociarse fiebre, anorexia y astenia, así como manifestaciones derivadas de un eventual compromiso mielorradicular $(1,18)$. Estas últimas tienden a ser más frecuentes entre las EIT a causa de una mayor demora diagnóstica, favorecida por un curso más indolente. Así, mientras en las EINT, el intervalo entre el inicio de los síntomas y el diagnóstico es habitualmente inferior a las 8 semanas, en las EIT se sitúa entre las 12 y las 24 semanas $(1,14,16)$. Las complicaciones neurológicas en el curso de las EI incluyen radiculopatías aisladas o múltiples, síndrome de la cola de caballo, paraparesia, paraplejías e incluso tetraplejia por compresión medular cervical $(1,7,9,18)$. El compromiso radicular es más frecuente cuando existe extensión paraespinal del proceso infeccioso, mientras que la compresión medular es más probable cuando se desarrolla un absceso epidural (7,23). La fiebre es más habitual en las EINT y puede sobrepasar los $38{ }^{\circ} \mathrm{C}$ en alrededor del $60 \%$ de los pacientes $(1,7)$.

La mayoría de las EI afectan sólo a un segmento raquídeo, siendo la columna lumbar y las últimas vértebras dorsales las más frecuentemente involucradas, tanto en las EIT $(1,14)$ como en las EINT $(1,4,7,15)$. En nuestros pacientes, las EIT afectaron predominantemente el segmento dorsal, mientras que las EINT, a igual que lo recogido en otras series $(4,7)$, se localizaron con mayor frecuencia en el segmento lumbar. La EI cervical supone menos del $10 \%$ de los casos y excepcional- 
mente cursa con formación de abscesos retrofaríngeos $(24,25)$ o con afectación atlo-axoidea. Aunque lo habitual es la afectación de un único segmento móvil (dos vértebras y el disco interpuesto entre ellas), es posible la extensión a otras vértebras contiguas, sobre todo en la EIT $(14,26)$. Los focos infecciosos en vértebras y discos pertenecientes a distintos segmentos móviles o EI politópicas, son raras $(<10 \%$ casos $)(2,7)$.

Las EI suelen cursar con elevación de la VSG y de los reactantes de fase, como la proteína $\mathrm{C}$ reactiva. En general, sus valores tienden a ser más elevados en las EINT (27). La leucocitosis es poco frecuente, sobre todo en las EIT. Aunque la intradermorreacción tuberculínica (PPD) es positiva en la práctica totalidad de las EIT, la elevada tasa de infección tuberculosa en nuestra comunidad autónoma reduce su interés como prueba de orientación diagnóstica. Los hemocultivos poseen una rentabilidad próxima al $50 \%(1,4,18)$, pudiendo ser superior en presencia de fiebre (7). Las radiografías simples de la columna vertebral suelen mostrar disminución del espacio discal, irregularidad de las plataformas epifisarias adyacentes al mismo, lesiones osteolíticas, con o sin esclerosis reactiva y signos sugestivos de absceso paravertebral. No existen diferencias significativas entre los hallazgos en ambos grupos etiológicos, aunque se ha considerado que las EIT tienden a producir lesiones osteolíticas y abscesos paravertebrales de mayor tamaño (27), hechos atribuibles a un más largo curso clínico previo al diagnóstico $(1,14,18,27)$. Para que las lesiones óseas resulten visibles en las radiografías simples se requiere un periodo mínimo de 2-3 semanas, que puede ser muy superior en las EI por microorganismos de baja virulencia; así, en nuestros cuatro pacientes con EINT (18\%) que no presentaban alteraciones radiográficas en el momento del diagnóstico, el tiempo transcurrido desde el inicio de los síntomas había sido inferior a 15 días. Las radiografías simples de tórax muestran lesiones pleuropulmonares en alrededor de la mitad de los pacientes con EIT, pero también pueden observarse signos de derrame pleural en un escaso número de pacientes con EINT dorsal, como ocurrió en uno de nuestros enfermos con EI D8-D9 por Staphylococcus aureus, en quien coexistía un derrame pleural izquierdo estéril. Dicho derrame sería la consecuencia de la irritación pleural inducida por la proximidad de la colección purulenta paravertebral. No obstante, también se ha descrito la aparición de verdaderos empiemas pleurales $(28,29)$. La resonancia magnética es la técnica de mayor rentabilidad en el diagnóstico por la imagen (Fig. 3), ya que muestra alteraciones precoces en la señal del disco y cuerpos vertebrales afectados (hiposeñal en las secuencias ponderadas en T1 e hiperseñal en las ponderadas en T2), siendo, al mismo tiempo, capaz de delimitar con precisión la extensión del proceso a partes blandas intrarraquídeas, como los abscesos epidurales, y extrarraquídeas, como los abscesos paravertebrales (30). La tomografía computarizada, aunque algo menos sensible, resulta especialmente útil para el diagnóstico, puesto que permite guiar las biopsias con trocar o las punciones aspirativas. Las gammagrafías óseas con $\mathrm{Tc}^{99 \mathrm{~m}} \mathrm{O}$ con Galio ${ }^{67}$ también poseen una alta sensibilidad, pero son menos específicas y no proporcionan información anatómica detallada. No obstante, son de utilidad cuando se sospechan otros focos de osteomielitis o en las EI multifocales (7).

La afectación de las leptomeninges en forma de epiduritis o abscesos epidurales puede asociarse a compromiso medular o mielorradicular. Por su parte, aunque pueden tener otros orígenes, casi la mitad de los abscesos epidurales se producen

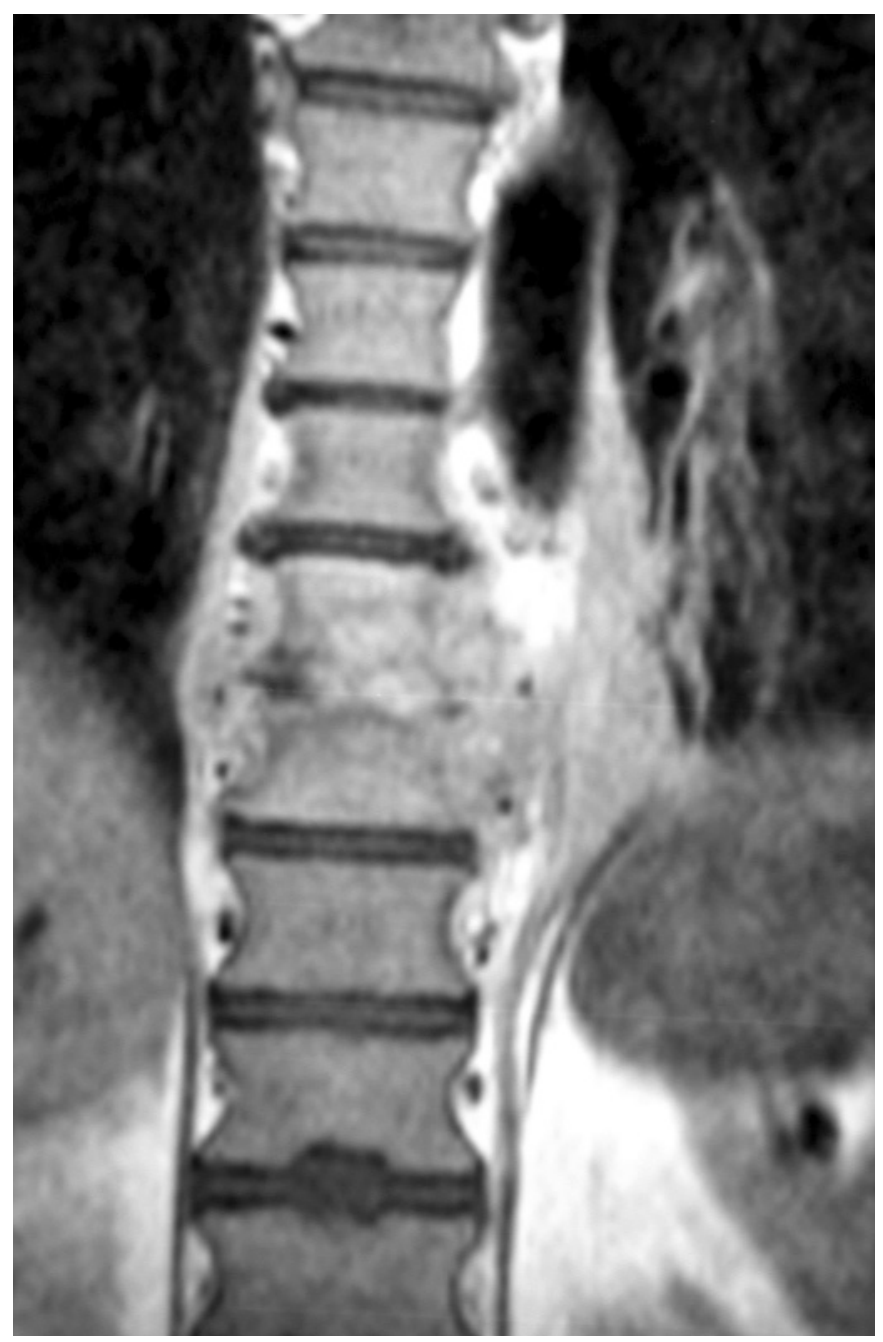

Fig. 3. Imagen coronal de resonancia magnética, ponderada en T2, correspondiente a uno de los pacientes con espondilodiscitis estafilocócica dorsal (D8-D9). Puede observarse la desaparición del espacio discal, las erosiones de los cuerpos vertebrales y la extensión a partes blandas adyacentes. El enfermo presentaba un empiema pleural izquierdo secundario.

como extensión de un foco infeccioso situado primariamente en el disco o en el cuerpo vertebral (31). La aparición de una meningitis séptica como complicación de una EI es rara y comporta un mayor riesgo vital (32).

La rentabilidad bacteriológica (tinciones de auraminarodamina o Ziehl-Neelsen y cultivos en medio Löwestein-Jensen) de las muestras de tejido o pus en las EIT oscila entre el $47 \%$ y el $78 \%$ debido a la escasa densidad de bacilos tuberculosos en las muestras de tejido o pus $(14,18)$. La existencia de granulomas caseificantes en las biopsias tisulares se considera suficiente para establecer el diagnóstico en un contexto clínico compatible, lo mismo que la positividad de los estudios microbiológicos en muestras alejadas del foco óseo, puesto que casi la mitad de los enfermos con EIT presenta tuberculosis extraesquelética activa, sobre todo pulmonar y pleural. Además, en la mayoría de las ocasiones, estas lesiones tuberculosas son más fácilmente accesibles que la propia EIT.

Más del $95 \%$ de las EINT están producidas por sólo un microorganismo, cuya naturaleza varía según la edad de los 
enfermos y los factores predisponentes $(6,9)$. El $64 \%$ de las EINT recogidas en nuestra serie fueron producidas por Staphylococcus aureus, seguidas por el género Streptococcus $(28,6 \%)$. No obstante, los datos obtenidos en otros estudios muestran que las bacterias gramnegativas van adquiriendo importancia creciente, sobre todo en los pacientes de más edad (1,5-7). En el 2-6\% de los enfermos la EINT se asocia a endocarditis, situación en la que predomina la etiología estreptocócica y en la que la puerta de entrada de los microorganismos suele ser bucodentaria o digestiva (33). A diferencia de otras series, no hemos encontrado EI por Brucella, lo que atribuimos al tipo de población asignado a nuestro centro, mayoritariamente urbana, con actividades no relacionadas con la ganadería y en la que es excepcional el consumo de productos lácteos no pasteurizados. Tampoco hallamos EINT producidas por hongos, aunque su frecuencia está aumentando en relación con el empleo de inmunosupresores y antibióticos de amplio espectro en pacientes trasplantados y afectados por neoplasias o enfermedades autoinmunes.

Las nueve EINT posquirúrgicas supusieron el 0,47\% de las intervenciones sobre la columna vertebral realizadas durante el periodo 1993-2003, porcentaje muy inferior al $3,7 \%$ recogido por Rohde y cols. (34) en 1642 discectomías lumbares. En siete (78\%) de dichas EINT el agente etiológico fue Staphylococcus aureus. No obstante, cada vez se comunican más casos producidos por gramnegativos y por microorganismos inhabituales $(2,35)$.

El tratamiento de las EI es fundamentalmente médico, debe instaurarse lo más precozmente posible, y se basa en reposo, la colocación de órtesis de descarga del segmento raquídeo afectado y la antibioticoterapia adecuada en cada caso. En las EIT, exceptuando resistencia o hipersensibilidad a alguno de los fármacos tuberculostáticos, se suele utilizar la misma combinación que para la tuberculosis pulmonar (isoniazida, rifampicina y etambutol durante tres meses, seguido de isoniazida y rifampicina durante otros seis meses). Por lo general, en las EINT la antibioticoterapia se inicia por vía intravenosa durante un periodo de 4-6 semanas y, posteriormente, se completa por vía oral. Los antibióticos deben seleccionarse según antibiograma y penetración ósea, pero la duración definitiva del tratamiento sigue siendo objeto de debate. A diferencia de la nuestra, muchas series incluyen pacientes en los que no se consiguió aislar el microorganismo causal, por lo que fueron tratados de manera empírica, valorándose posteriormente la respuesta clínica, biológica y radiológica (3). Las medidas ortésicas (minervas cervicales, corsés dorsolumbares o lumbares) constituyen un importante complemento a la hora de descargar el segmento afectado y proporcionarle estabilidad durante el proceso de reparación ósea. La cirugía, aparte de la toma de muestras tisulares para el diagnóstico etiológico cuando otros medios han fracasado, queda supeditada a la presencia abscesos paravertebrales voluminosos, compromiso mielorradicular o grave inestabilidad vertebral, lo que ocurre en el $10-20 \%$ de los pacientes $(3,36,37)$.

El porcentaje de recidivas en las EINT es variable según las series, oscilando del 3 al 20\% $(7,18,20,38)$. En nuestra casuística la única recidiva se objetivó en un paciente con EIT. En la actualidad, la mortalidad derivada de las EI es inferior al 5\%, aunque la prevalencia de secuelas como deformidad, dolor local y déficit neurológicos es relativamente elevada, situándose entre el 8 y el $41 \%$ de los pacientes, según etiología, localización y retraso en el diagnóstico $(1,7,18)$.

\section{Bibliografía}

1. Perronne C, Saba J, Behloul Z, Salmón-Céron D, Leport C, Vildé JL, Khan MF. P. Pyogenic and tuberculous spondylodiskitis (vertebral osteomielitis) in 80 adults patients. Clin Infect Dis 1994; 19: 746-50.

2. Fiter J, Gómez C, Miquel J. Espondilodiscitis infecciosa. Perspectiva actual de un viejo problema. Rev Esp Reumatol 1999; 26: 91-9.

3. Belzunegui J, González C, Figueroa M, del Val M, Erausquin C, Rodríguez-Valverde $\mathrm{V}$. Infectious spondylodiscitis: a revision of 98 cases. $\mathrm{Br}$ J Rheumatol 1996; 35 (Supl. 1): 166

4. García-Vadillo JA, López-Robledillo JC, Díaz-González F, Verdejo J, Umbría A, García-Vicuña R. Espondilodiscitis infecciosa. Estudio de 30 casos. Rev Esp Reumatol 1991; 18: 123-6.

5. Sapico FL. Microbiology and antimicrobial therapy of spinal infections. Orthop Clin North Am 1996; 27: 9-13.

6. Carragee EJ. Pyogenic vertebral osteomyelitis. J Bone Joint Surg 1997; 79 (Am): 874-80.

7. Nolla JM, Ariza J, Gómez-Vaquero C, Fiter J, Bermejo J, Valverde J, et al. Spontaneous pyogenic vertebral osteomyelits in nondrug users. Sem Arthritis Rheum 2002; 31: 271-8.

8. Sapico FL, Montgomerie JZ. Vertebral osteomyelitis. In Norden C, ed. Bone and Joint infections, new perspectives. Philadelphia. W B Saunders, 1990; pp: 539-50.

9. Torda AJ, Gottlieb T, Bradbury R. Pyogenic vertebral osteomyelitis: analysis of 20 cases and review. Clin Infect Dis 1995; 20: 320-8.

10. Segal BH, Sneller MC. Infectious complications of immunossuppresive therapy in patients with rheumatic diseases. Rheum Dis Clin North Am 1997; 23: 219-37.
11. Azzan NI, Tammawy M. Tuberculous spondylitis in adults: diagnosis and treatment. Br J Neurosurg 1988; 2: 85-91.

12. Buchelt M, Lack W, Kutschera HP, Katterschafta T, Kiss H, Scheneider B, Kotz R. Comparison of tuberculous and pyogenic spondylitis. An analysis of 122 cases. Clin Orthop 1993; 296: 192-9.

13. Al-Muhin FA, Ibrahim EM, El-Hassan AY, Moharram HM. Magnetic resonance imaging in tuberculous spondylitis. Spine 1995; 20: 2.28792.

14. Pertuiset E, Beaudreuil J, Lioté F, Horusitzky A, Kemiche F, Richette P, et al. Spinal tuberculosis in adults. A study of 103 cases in a developed country, 1980-1994. Medicine (Balt) 1999; 78: 309-20.

15. Krogsgaard MR, Wagn P, Bengtsson J. Epidemiology of acute vertebral osteomyelitis in Denmark: 137 cases in Denmark 1978-1982 compared to cases reported to the National Patient Register 1991-1993. Act Orthop Scand 1998; 69: 513-7.

16. Hayes AJ, Choksey M, Barnes N, Sparrow OCE. Spinal tuberculosis in developed countries: difficulties in diagnosis. J R Coll Surg Edinb 1996; 41: 192-6.

17. Fiter J, Nolla JM, Gómez-Vaquero C, Ros S, Ariza J, Roigt-Escofet D. Septic spondylodiscitis induced by pyogenic germs in patients without addiction to intravenous drugs. Br J Rheumatol 1996; 35 (Supl. 1): 165.

18. Colmenero JD, Jiménez-Mejías ME, Sánchez-Lora FJ, Reguera JM Palomino-Nicas J, Martos F, et al. Pyogenic, tuberculous, and brucellar vertebral osteomyelitis: A descriptive and comparative study of 219 cases. Ann Rheum Dis 1997; 56: 709-15.

19. Rouauld J P, Caroit M. Spondilodiscites d'inoculation. Rev Rhum Mal Osteoartic 1981; 48: 107-11. 
20. Ozuna RM, Delamarter RB. Pyogenic vertebral osteomyelitis and postsurgical disc space infections. Orthop Clin North Am 1996; 27: 87-94.

21. Honan M, White GW, Eisenberg GM. Spontaneous infectious discitis in adults. Am J Med 1996; 100: 85-9.

22. Olivé A, Pérez R, Tena X, Guasch I, Romeu J, Clotet B. Musculoskeletal manifestations of HIV infection. Br J Rheumatol 1996; 35 (Supl 1): 167.

23. Del Curling O Jr, Grower DJ, McWhorter M Mc. Changing concepts in spinal epidural abscess: A report of 29 cases. Neurosurgery 1990; 27: 185-92.

24. Faidas A, Ferguson J V Jr, Nelson J E, Baddour L M. Cervical vertebral osteomyelitis presenting as a retropharingeal abscess. Clin Infect Dis 1994; 18: 992-4.

25. López J, Eres N, Fernández X, Buti M. Celulitis por Streptococcus pneumoniae asociada a espondilodiscitis tras cuadro de otitis media supurativa. Enf Infecc Microbiol Clin 1997; 15: 171-2.

26. Vadilo M, Corbella X, Mascaró J, Niubó R. Espondilitis tuberculosa politópica como presentación de una tuberculosis miliar. Enferm Infecc Microbiol Clin 1995; 13: 123-4.

27. Gómez N, Martínez C, Sopeña B, Arasa X, Barrio E. Estudio comparativo entre el mal de Pott y espondilodiscitis no tuberculosas. Rev Clin Esp 1989; 184: 72.

28. Mateos A, Flórez J, Monte R. Derrame pleural asociado a osteomielitis vertebral. Arch Bronconeumol 1995; 31: 430-1.

29. Jari S, El-Gamed A, Meadows T H, Campbell C. Spinal osteomyelitis presenting with a life-threatening pleural empyema. Spine 1996; 21: 2.806-8.
30. Maiuri F, Iaconeta G, Gallicchio B, Manto A, Briganti F. The spondylodiskitis: A clinic and magnetic resonance diagnostic. Spine 1997; 22: 1741-6.

31. Del Curling O Jr, Gower D J, McWhorter J M. Changing concepts in spinal epidural abscess: a report of 29 cases. Neurosurgery 1990; 27: 185-192.

32. Rodríguez M, Fernández L, López G, Zarzalejos J M, Rozas P. Espondilodiscitis por gérmenes piógenos. Estudio prospectivo de 15 casos. Rev Esp Reumatol 1992; 19: 258-62.

33. Marsal S, Castro-Guardiola A, Clemente C, Tornos P, Pahissa A, Follonosa V et al. Streptococcus bovis endocarditis presenting as acute spondylodiscitis. Br J Rheumatol 1994; 33: 403-4.

34. Rohde V, Meyer B, Schaller C, Hassler W E. Spondylodiscitis after lumbar discectomy: Incidence an a proposal for prophylaxis. Spine 1998; 23: 615-20.

35. Bontoux D, Codello L, Debiais F, Lambert de Cursay G, Azais I, Alcalay M. Espondylodiscites infectieuses. Analyse d'une série de 105 cas. Rev Rhum Mal Osteoartic 1992; 59: 401-7.

36. Emery SE, Chan DPK, Woodward HR. Treatment of hematogenous pyogenic vertebral osteomyelitis with anterior debridement and primary bone grafting. Spine 1989; 14: 284-91.

37. Özdemir HM, Kemal A, Ögün T. The role of anterior spinal instrumentation and allograft fibula for the treatment of Pott disease. Spine 2003 28: 474-9.

38. Patzakis MJ, Rao S, Wilkins J, Noore TM, Harvey PJ. Analysis of 61 cases of vertebral osteomyelitis. Clin Orthop 1991; 264: 178-83. 